\title{
Acyclic diene metathesis polymerization and precision polymers
}

\author{
Nicolas F. Sauty $\cdot$ Lucas Caire da Silva \\ Michael D. Schulz $\cdot$ Chip S. Few $\cdot$ Kenneth B. Wagener
}

Received: 10 January 2014/ Accepted: 27 January 2014/ Published online: 19 February 2014

(c) The Author(s) 2014. This article is published with open access at Springerlink.com

\begin{abstract}
The history of and major advances in the acyclic diene metathesis (ADMET) reaction are described. Because precise branch identity and frequency can be achieved by ADMET polymerizations of symmetrical $\alpha, \omega$ dienes, polyethylenes with precisely spaced alkyl branches of specified length have been prepared. Investigations of their morphologies and thermal properties have provided valuable insight into the behavior of polyethylene. ADMET preparation of ethylene copolymers and telechelic oligomers, as well as the properties of these materials, is also discussed.
\end{abstract}

Keywords ADMET polymerization - Olefin metathesis · Precision polymers · Polyethylene

\section{Introduction}

The development of olefin metathesis has led to remarkable achievements in organic and polymer chemistry. From allowing facile synthesis of complex natural products to producing intricate polymer architectures, olefin metathesis has had an undeniable impact on organic and polymer synthesis. There are two distinct modes of metathesis polymerization: ring-opening metathesis polymerization (ROMP) and acyclic diene metathesis (ADMET) polymerization [1, 2] (Fig. 1). The development of the ADMET reaction is the subject of this review.

N. F. Sauty - L. C. da Silva - M. D. Schulz •

C. S. Few · K. B. Wagener $(\square)$

The George and Josephine Butler Polymer Research Laboratory,

Department of Chemistry and Center for Macromolecular

Science and Engineering, University of Florida,

Gainesville, FL 32611-7200, USA

e-mail: wagener@chem.ufl.edu

\section{Historical background}

The development of well-defined, efficient olefin metathesis catalysts (Fig. 2) was paramount to the development of the ADMET reaction. Although classical olefin metathesis catalysts were effective in some cases, the exact nature of the catalytically active species was not known. Early work by Grubbs [3-7] and Schrock [8-12] produced some of the first spectroscopically identifiable olefin metathesis catalysts.

The introduction of Schrock's catalysts (e.g., [Mo]1) enabled significant progress in olefin metathesis and its use in organic and polymer synthesis. However, these catalysts are sensitive to air, water, and some polar functional groups, and this sensitivity has limited their application in some cases. To address this issue, late transition metal catalysts were developed both by Schrock [13] and Grubbs [14]. Of these, the Grubbs ruthenium-based catalysts have been particularly successful. The first generation of these ruthenium complexes was reported in 1992 and 1993 [1518]. Although first-generation ruthenium catalysts such as [Ru]1 have less activity than the Mo-based Schrock catalysts, the Grubbs' complexes are more tolerant to moisture, oxygen, and polar functional groups. A second-generation of ruthenium catalysts, principally $[\mathbf{R u}] \mathbf{2}$, resulted from the replacement of a phosphine with an $N$-heterocyclic carbene (NHC) ligand. These catalysts are very reactive in olefin metathesis reactions and possess greater functional group tolerance than the first-generation ruthenium catalysts [1921]. The activity of some second-generation ruthenium catalysts is comparable to the activity of [Mo]1 [22]. In 1999, a new class of olefin metathesis catalysts with an isopropoxy group on the benzylidene moiety, $[\mathbf{R u}] \mathbf{3}$, was reported by Hoveyda and coworkers [23]. Soon thereafter, the second-generation Hoveyda-Grubbs catalysts were 


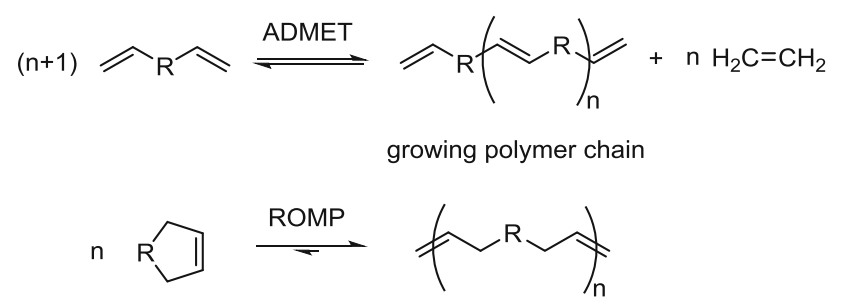

Fig. 1 The ADMET reaction

reported, all incorporating an NHC ligand ([Ru]4 is one example). These catalysts are very effective for many different olefin metathesis reactions [22, 24].

These and other catalyst developments [25] allowed ADMET to be a useful and feasible reaction. The Wagener group began investigating unconjugated $\alpha, \omega$-diene polycondensation, later known as ADMET polymerization, using the $\mathrm{WCl}_{6} / \mathrm{EtAlCl}_{2}$ catalyst system in the late $1980 \mathrm{~s}$ [26]. These reactions resulted in a combination of soluble oils and intractable solids. It was determined that the oil was a low molecular weight polymer-the result of olefin metathesis. It was believed that the insoluble product resulted from a vinyl addition reaction, which, in competition with metathesis, would result in a crosslinked polymer, but the solid product was not rigorously characterized. In order to test this hypothesis, Wagener and coworkers reacted $\mathrm{WCl}_{6} / \mathrm{EtAlCl}_{2}$ with styrene. The predicted product of olefin metathesis for this reaction would be stilbene; however, the reaction also yielded polystyrene. This result demonstrated that vinyl addition and olefin metathesis are competing reactions when these catalyst systems are used. To achieve high polymer, the competing vinyl addition reaction must be eliminated. Serendipitously, this work coincided with the report of catalyst [Mo]1. The concurrence of these advances enabled ADMET to be applied as a means of synthesizing high molecule weight polymer.

\section{The ADMET reaction}

Acyclic diene metathesis polymerization is an equilibrium step-growth condensation of $\alpha, \omega$-dienes promoted by a suitable metathesis catalyst. The reaction produces a growing unsaturated polymer chain that retains the symmetry and functionality of the monomer.

Over the years, monomer design and the development of suitable catalysts have opened the way to a variety of welldefined polymer structures which are not readily achievable by other common techniques. The ADMET polymerization proceeds via a metal-carbene mechanism, in which the metal methylidene is the true catalyst formed during the polymerization cycle (Fig. 3). This is the same metal-carbene mechanism operating in cross-metathesis [27]. Coordination of the metallic center of the catalyst with a terminal olefin from either the monomer or polymer is followed by a reversible $2+2$ cycloaddition to produce a metallocyclobutane intermediate. This undergoes further reversion leading to either a productive or a non-productive pathway. Chain growth is promoted by the productive metathesis pathway resulting from cycloreversion, which will not restore the coordinating olefin and free catalyst. This pathway is characterized by production of ethylene, which should be removed from the reaction mixture in order to drive the equilibrium towards chain growth. ADMET polymerization affords PDIs around 2, which are typical for step-growth polymerization. Also, molecular weights up to $50,000 \mathrm{~g} / \mathrm{mol}$ can be achieved, depending primarily on catalyst life time, monomer purity, and reaction conditions. It is fair to say that catalyst compatibility and monomer design/purification are the main challenges to be overcome in order to achieve high polymer with welldefined primary structure.

ADMET polymerization usually requires relatively long reaction times in order to guarantee high conversion and consequently high molecular weight polymers. Because some reactions require days to be completed, catalyst lifetime can be a limiting parameter. Catalyst lifetime depends primarily on the rate of deactivation by catalyst poisoning and can be a major problem if a compatible metathesis catalyst is not available. Grubbs' rutheniumbased and Schrock's molybdenum-based catalysts [28] are among the most used catalysts for ADMET. Grubbs' firstgeneration catalyst is very common due to its high tolerance towards moisture, oxygen and coordinating functional groups, even though its reactivity is not as high as Schrock's catalyst [29]. The highly reactive Schrock's catalyst

Fig. 2 Five examples of welldefined olefin metathesis catalysts
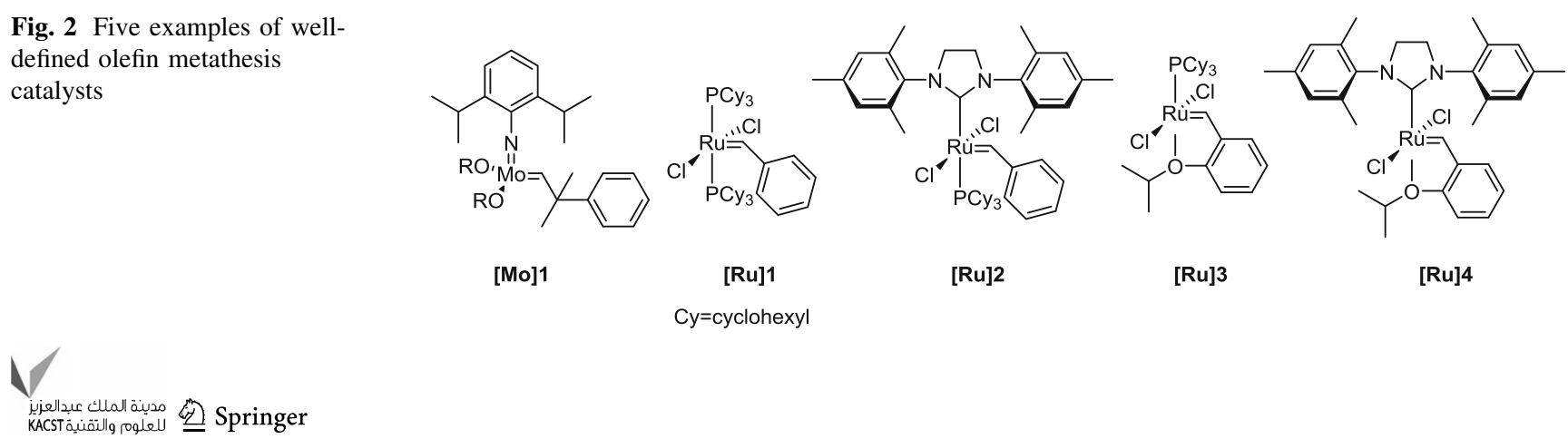


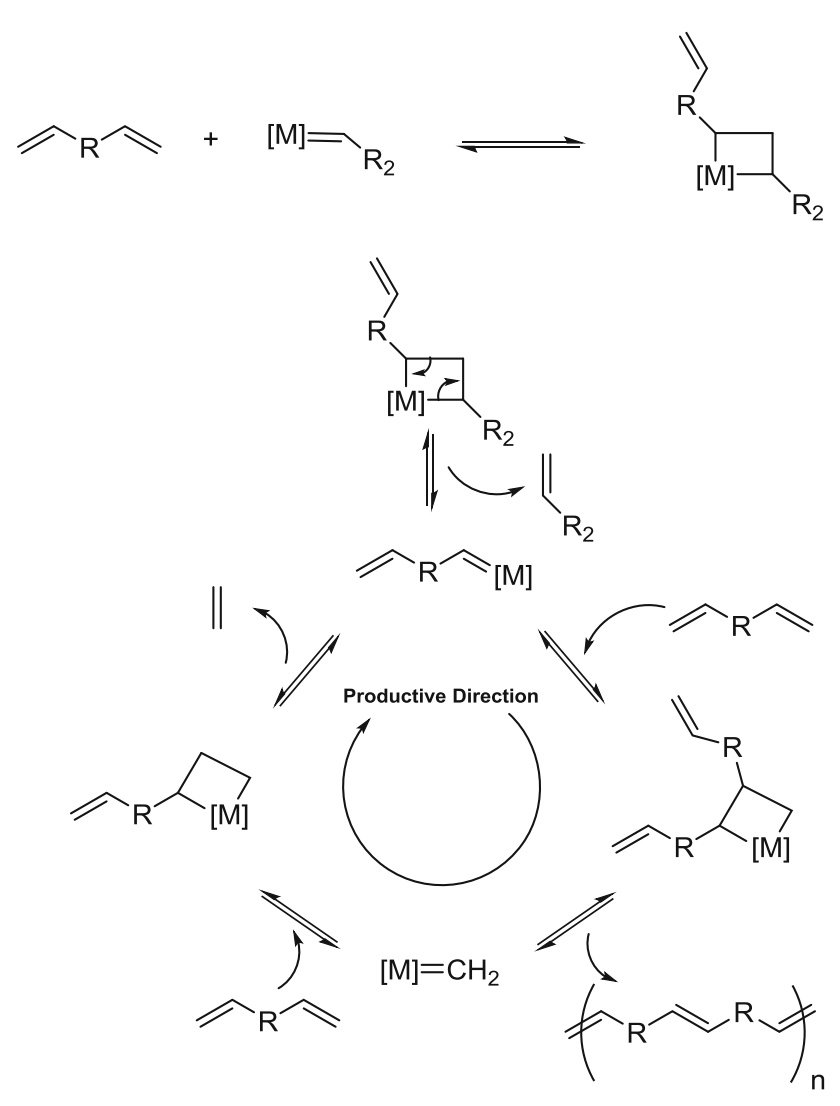

Fig. 3 The ADMET mechanism

is much more sensitive and requires careful handling and extremely dry and pure monomers. The high oxidation state of the molybdenum metal center makes the catalyst highly vulnerable to polar functional groups, water and oxygen. Traces of impurities can deactivate the catalyst rendering very poor conversion/molecular weights.

Even though there are a large number of metathesis catalysts available, many are known to promote isomerization, which ultimately results in loss of control over the polymer primary structure. Grubbs' first generation and Schrock's catalysts are known to promote minimal isomerization under regular ADMET reaction conditions, while studies on Grubbs' second generation, as well as other ruthenium-based metathesis catalysts, shows significant isomerization under similar conditions [30, 31]. Isomerization is a drawback, especially when the choice of a catalyst is limited by monomer functionality. Fortunately, several methods are available to minimize isomerization, usually by use of special additives such as benzoquinones [32, 33] or boron-containing Lewis acids [34].

Virtually any $\alpha, \omega$-diene can be polymerized via ADMET if a suitable catalyst is available that tolerates the desired monomer functionality. The mechanism governing metathesis ensures control over primary structure based on retention of monomer symmetry/asymmetry in the final polymer. Saturation of the internal olefins of the unsaturated ADMET polymer eliminates the cis/trans distribution, allowing for polymers with well-defined primary structures. Tacticity in ADMET polymers is not yet fully controlled and is being currently addressed by the Wagener group.

ADMET polymerization is ideally carried out in the bulk with monomer-to-catalyst ratios typically in the range of 100-500:1 under high vacuum. The increased viscosity during polymerization is overcome by performing the reaction at temperatures ranging from 40 to $75{ }^{\circ} \mathrm{C}$ under constant stirring. Where solubility is not an issue, solvents can be used to maintain low viscosity levels, as long as the catalyst is not deactivated in solution. Plenio and coworkers showed that high-boiling solvents such as 1,2dichlorobenzene can be used in ADMET polymerization when a slight vacuum is desirable [35]. More recently, Simocko et al. [36] demonstrated that ionic liquids are excellent media to perform ADMET polymerizations. Grubbs' catalyst is usually employed when solvents need to be used, given its higher tolerance towards contaminants and polar functional groups. During the polymerization, removal of ethylene condensate can be achieved by applying high vacuum, or by purging the reaction vessel with an inert gas, in order to drive the reaction to completion. This can be achieved by applying high vacuum or by purging the reaction vessel with an inert gas in order to push the reaction to completion. Reaction progress can be easily followed by comparing the relative ${ }^{1} \mathrm{H}$ NMR signal intensities of the terminal olefin on the monomer and internal olefin of the resulting unsaturated polymer.

\section{Modeling polyethylene}

Polyethylene (PE) is the most produced polymer and still dominates the polymer market because of its low cost, versatility, and ease of manufacture [37]. Conventional industrial methods inherently afford polyethylenes with random branches of various lengths along the polymer chain, and this branching greatly affects $\mathbf{P E}$ properties [38]. Over the years, considerable effort has been devoted to controlling branching and ideally to producing linear polyethylene. In addition, copolymerization of ethylene with polar vinyl monomers often leads to a higher branching content in conjunction with the low incorporation of the polar entity in the polymer backbone. This eventually produces a gradient polymer rather than a statistical polymer.

ADMET polymerization of symmetrical diene monomers is a remarkable tool to afford flawless and impeccably regular polyolefins. Subsequent hydrogenation produces polyethylene copolymers possessing a functionality (either within the polymer backbone or side branching) at 
Fig. 4 Monomer synthesis

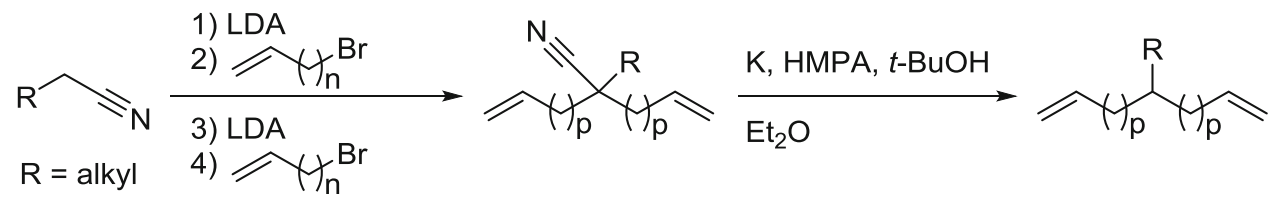

Fig. 5 Synthesis of precision alkyl-branched polyethylene

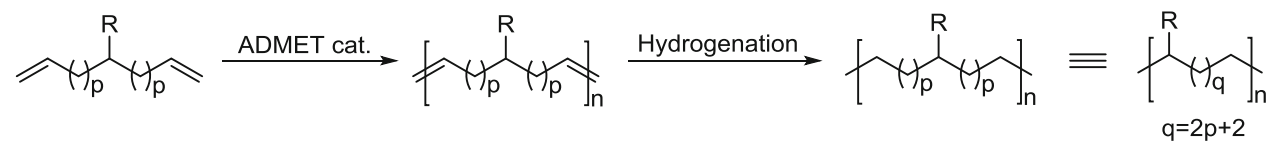

perfectly unvarying intervals (i.e., separated by a constant number of methylene spacers). Depending on the catalyst, molecular weights $\left(M_{\mathrm{n}}\right)$ of ADMET polyethylene copolymers usually range from 5,000 to $50,000 \mathrm{~g} / \mathrm{mol}$. These $M_{\mathrm{n}} \mathrm{s}$ are sufficient to be accepted as polyethylene models, given that the entanglement molecular weight of linear polyethylene is about $1,000 \mathrm{~g} / \mathrm{mol}$ [39].

ADMET polymerization offers a unique way to model branched polyethylene, as well as any copolymers of ethylene and $\alpha$-alkenes $\left(\mathrm{C}_{n} \mathrm{H}_{2 n}\right)$. A two-step route involving a dialkylation of a nitrile intermediate followed a reductive decyanation [40] has been used to prepare a collection of symmetrical alkyl-branched $\alpha, \omega$-dienes (Fig. 4).

Subjecting these monomers to ADMET polymerization and subsequent exhaustive hydrogenation afforded polyethylenes with precise placement (every $n$th carbon) of alkyl branches on their backbones (Fig. 5).

Conversely, statistical placement of alkyl branches can be obtained by copolymerization of the aforementioned symmetrical monomer with a linear $\alpha, \omega$-diene (typically 1,9-decadiene) [41]. Precision alkyl-branched polyethylenes were found to possess enhanced thermal properties due to higher crystallinity, leading to sharper melting transitions at lower temperatures and greater heats of fusion. In precision polymers, two factors determine the thermal properties and morphologic parameters of these materials: the branch identity and the branch frequency. The crystalline structures have been studied using wide and small angle X-ray scattering (WAXS and SAXS), transmission electron microscopy (TEM) and infrared (IR) spectroscopy. Morphology is greatly influenced by the alkyl branch size and spacing.

Regularity in primary structure predictably translates to uniformity in the form of narrow lamellar thickness distributions in polyethylene containing precisely spaced alkyl branches [42]. The lamellar thickness corresponds to the distance between branch sites in these materials, which suggests that the chains are folding at the branch sites, with the branches residing at the interface of the lamellae [43]. As a consequence of the narrow lamellar thickness distribution, ADMET polymers have a narrower melt temperature range compared to other polyethylenes.

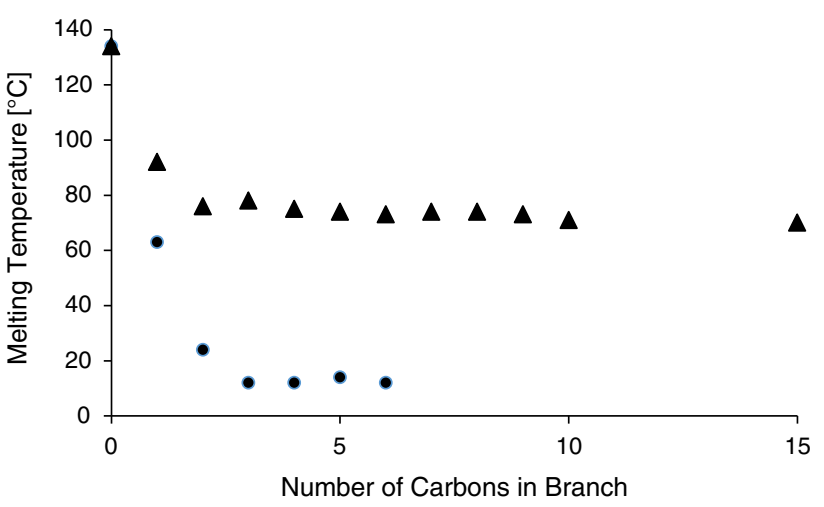

Fig. 6 The effect of alkyl branch size on $T_{\mathrm{m}}$ can be seen for ADMET polyethylenes with branches on every 21st (filled circle) and 39th (filled triangle) carbon along the polymer backbone

Multifarious branch sizes, ranging from methyl to pentadecyl, have been incorporated in a precise fashion on the polyethylene backbone. It was discovered that the resulting fusion temperatures were highly dependent on the nature of the branching (Fig. 6). As a general rule, the melting temperature dwindles with longer alkyl branches. The effect of alkyl branch size on the melting temperature can be seen for ADMET polyethylenes with branches on every 21st [44] and 39th [45] carbon. Polymers with branches excluded from the lamellae exhibit similar fusion temperatures. This expulsion of alkyl chains from the lamellae is affected by defect size, as ADMET polymers bearing smaller alkyl groups (e.g., methyl) crystallize with a greater degree of branch inclusion. This is true of precision polymers of varying branch spacing, and the maximum included branch size decreases with increasing branch interval. Indeed, when the branch is placed on every 21 st carbon, methyl and ethyl branches are included in the unit cell and afford polymers displaying melting transitions at 63 and $24^{\circ} \mathrm{C}$, respectively. Conversely, polymers with branches larger than the maximum included branch size exhibit similar melting temperatures. In other words, the transition becomes independent of the branch identity with $T_{\mathrm{m}} \mathrm{s}$ of approximately $12{ }^{\circ} \mathrm{C}$. Similarly, when an alkyl defect is placed every 39th carbon, only a methyl branch can be included in the unit cell, and as a consequence, 


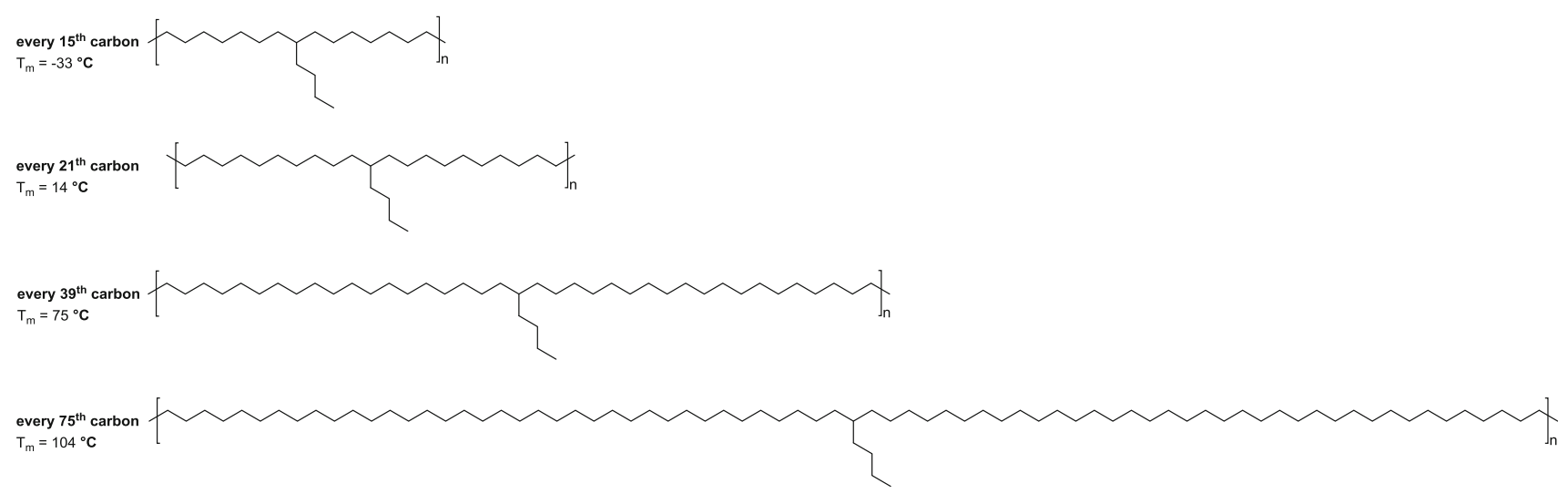

Fig. 7 Branch frequency: alkyl branch every $n$th carbon

similar melting temperatures are found for the polymers bearing longer branches.

Various branch frequencies have been investigated with the goal of diminishing the frequency (increasing the spacing) as much as possible. The general observation is that for a given branch identity, thermal transitions occur at higher temperatures with diminished frequencies. The Wagener group has synthesized butyl-branched polyethylenes in which the branch appears every 9th, 21st, 39th, and 75th [46] carbon (Fig. 7).

This has led to the discovery of a linear relationship between the branch frequency and fusion temperature of the aforementioned polymers. It also suggested a melting temperature of $136{ }^{\circ} \mathrm{C}$ for an infinitely low frequency which is quite close to that of linear ADMET polyethylene $\left(134{ }^{\circ} \mathrm{C}\right)$.

The distance between alkyl branches also has an effect on the crystalline lattice, as less frequent branching leads to more ordered structure. The extreme case, with no branching, leads to orthorhombic structure. The introduction of branches leads to less-ordered crystalline structures, of which there are several examples in the literature [47, 48]. When precisely spaced alkyl groups are placed every 7th carbon or closer, the resulting polymers are amorphous.

The morphological effects described in the preceding paragraphs can be further understood by considering the crystallization behavior of representative polymers. As is the case in polyethylenes synthesized by other means, ADMET polyethylenes crystallize through the formation of a hexagonal mesophase, which expands as the chains slide past one another in the all trans conformation until the equilibrium lamellar thickness is reached [49]. While crystallization of longer methylene sequences occurs before shorter sequences in metallocene PE, forcing shorter methylene sequences into the amorphous phase, all methylene sequences are of the same length in ADMET polyethylene, allowing crystallites of equilibrium lamellar thickness to form from the transient hexagonal mesophase [50].
By varying the size and interval of alkyl branches on polyethylene, the resulting morphological effects are delineated. Uniform ethylene sequences allow a metastable mesophase to form, at which point the chains can reposition themselves into an energetically stable morphology, resulting in a material with narrow lamellar thickness distribution. Depending on defect size, the branches may be contained within the lamellar stem in the case of smaller branches, or excluded to the surface of the crystallite in the case of longer branches. The lamellar thickness and crystalline lattice type depend on the distance between defects, with longer intervals leading to more ordered polymorphisms.

Beyond the thermal and morphologic elucidation of precision alkyl-branched polyethylene, molecular dynamics were probed utilizing deuterium quadrupolar echo NMR in the solid state. The ${ }^{2} \mathrm{H}-\mathrm{NMR}$ spectra of precision $\mathrm{CD}_{3}$-branched polyethylene were acquired for a wide range of temperatures [51, 52]. A $T_{1}$-fitting methodology was applied to deconvolute the spectral contributions of the amorphous and crystalline phases, allowing isolation of two distinctive dynamic behaviors. This is the consequence of a bi-exponential behavior of the spin-lattice relaxation in the $\mathrm{CD}_{3}$ branches. The amorphous phase exhibited a similar behavior to that of linear polyethylene [53]. Conversely, the crystalline domains displayed a harmonic liberational axial rotation reorientation model with a mean jump angle up to $40^{\circ}$ when approaching the melting transition. Various other deuterated precision polymers are being analyzed by deuterium quadrupolar echo methods with the intention of elucidating the dynamics of precision polymers.

\section{Ethylene copolymers}

The advent of ADMET polymerization not only promoted modeling of polyethylene, but also stimulated the understanding of numerous classes of polymers. Various 
Fig. 8 Synthesis of precision poly(ethylene-co-acrylic acid)

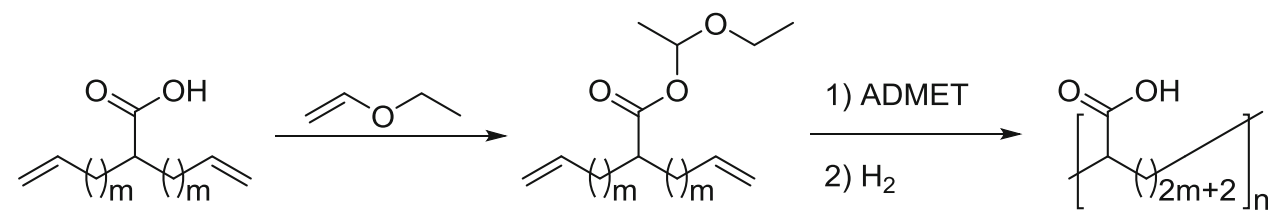

copolymers of ethylene and polar vinyl intermediates were scrutinized and compared to commercial samples. All syntheses relied on the ADMET polymerization of symmetrical $\alpha, \omega$-diene monomers followed by exhaustive hydrogenation to afford the desired polyethylene precision copolymers. Relevant examples are briefly described in this section.

Ethylene-vinyl acetate copolymers (EVA) are widespread materials, with hot-melt adhesives and plastic wraps being common applications [54]. Although free-radical copolymerizations usually produce gradient or block polymers, perfectly random copolymers are obtained from the polymerization of ethylene and vinyl acetate due to similar reactivities [55]. However, undesired and random branching is inherently promoted via a back-biting process, significantly altering the characteristics and performance of the copolymers. However, perfectly linear ethylene-vinyl acetate copolymers can be prepared by means of ADMET polymerization [56]. A number of monomers with various methylene run lengths ranging from 8 to 12 were subjected to polymerization and subsequent hydrogenation, furnishing copolymers with different vinyl acetate contents (acetate branches separated by 18-26 carbons). Commercial samples with similar vinyl acetate ratios were compared with the ADMET copolymers. Similar to the commercial copolymers, the precision polymers displayed a linear relationship between the melting temperature and vinyl acetate content, but with dissimilar slopes. A larger slope, underlining the precision factor, evidenced the greater order in precision polymers. With a similar approach, copolymers of ethylene and vinyl alcohol were made without difficulty.

While metathesis catalysts such as [Ru]1 can tolerate a variety of functional groups, functionalities such as primary or secondary amines, sulfides, or carboxylic acids deactivate the catalyst, thereby precluding preparation of high molecular weight materials (oligomers to about $3,000 \mathrm{~g} / \mathrm{mol}$ were produced). In the case of carboxylic acids, this problem can be circumvented by protecting the acid functionality. Various agents can be employed, but ethyl vinyl ether was found to be extremely convenient [57]. The resulting 1-ethoxyethyl ester, inert to ruthenium catalysts, is quantitatively cleaved during the hydrogenation of the resulting polyolefin (Fig. 8).

The ethylene-acrylic acid copolymers were found to be amorphous when only 8 or 14 methylene units separated two consecutive branching sites. Conversely, increasing the spacing to 20 methylenes resulted in a semicrystalline material displaying dimerized acid moieties in an orthorhombic lattice. Simple treatment of the copolymer with zinc reagents, produced a zinc-neutralized material containing $22 \%$ free acid and $66 \%$ zinc dicarboxylates. The zinc dicarboxylate components aggregated into a selfassembled structures adopting a cubic crystalline lattice, an unprecedented observation for ionomers having a purely hydrocarbon backbone [58].

Precision ethylene-vinyl halide copolymers have also been examined [59]. Contrary to many other systems, it was discovered that regardless of the halide identity (except iodide) and appearance frequency on the polymer backbone, the halogen atoms were included in the unit cell. This yielded an orthorhombic system in the case of fluorine, and triclinic systems in the case of the larger chlorine and bromine substituents.

Motivated by the recent advances in fuel cell membranes, precision polymers incorporating sulfonic [60] and phosphonic $[61,62]$ acids have been investigated for potential uses as proton carriers. As with carboxylic acids, the aforementioned functionalities must be protected prior to polymerization to prevent poisoning of the metathesis catalyst. While ethyl phosphonates could straightforwardly be deprotected post-hydrogenation, the sulfonate analogues have not led to comparable results. The significant insolubility of the poly(ethylene-co-vinylsulfonic acid)s promotes premature precipitation of the material and prevents complete deprotection. This problem is currently being addressed by the Wagener group. With sufficient spacing (20 methylene spacers or greater), the ethylene-vinylphosphonic acid copolymers show a semicrystalline behavior. Amorphous materials are promoted by shorter methylene segments.

Finally, among others, ADMET polymerization has been utilized to produce precision materials containing ether [63], silicon [64], or amine [65] moieties.

\section{Telechelic oligomers via ADMET}

Many telechelic oligomers (oligomers with functionalized end groups) have been synthesized by ADMET. These materials are often macromonomers used to synthesize larger polymers, and they have many other uses as well. Telechelic oligomers of polybutadiene (Fig. 9) have been prepared via the reaction of 1,5-hexadiene and a mono- or 
Fig. 9 Synthesis of telechelics via ADMET

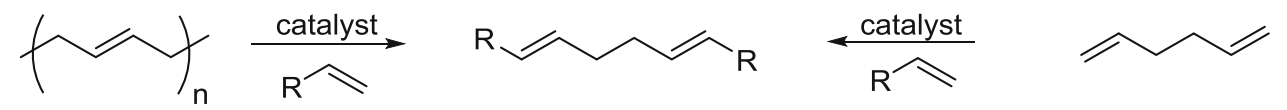

$\mathrm{R}=$ silane, ester, alkyl di-functional olefin $[66,67]$. ADMET polybutadiene may alternatively be achieved by the polymerization of 1,5hexadiene. This polymer can then be used to prepare telechelic oligomers by reacting with a suitable olefin. Sometimes the reaction is incomplete, producing telechelic oligomers. Additionally, polybutadiene produced by cationic polymerization can undergo ADMET depolymerization with acrylates to yield ester-terminated telechelics [68]. This approach was also used to produce a polyamide by depolymerizing polybutadiene with acryloyl chloride and subsequently reacting with 1,6-diaminohexane. Ester-end-capped polyethylene oligomers have been made by ADMET by reacting 1,9-decadiene and 9-decenyl acetate and subsequently hydrogenating the unsaturated units. Telechelic polyacetylenes have been synthesized by ADMET polymerization of 2,4-hexadiene with 1,2-disubstituted olefins [69]. Polyacetylene oligomers terminated with a variety of end groups, including silanes, alkyl groups, and esters were prepared with this method.

Hydrophobic, amorphous telechelic diols have also been synthesized by ADMET [70]. These materials could potentially be used in UV- and hydrolysis-resistant polyurethanes. The hydrocarbon backbone was produced by ADMET polymerization followed by hydrogenation of a gem-dimethyl substituted $\alpha, \omega$-diene, and this material was then endcapped with alcohol-containing monoenes with varied numbers of methylenes between the alcohol group and olefin. Some molecular weight control was achieved in the resulting telechelics, which had 2.0 functionality.

In another report, 1,9-decadiene was polymerized using [Ru]1 with epoxy-containing monoenes [71].The resulting telechelic polymer was then reacted with toluene diisocyanate resulting in a copolymer containing oxazolidone linkages. ADMET has been used to synthesize ester-terminated telechelics with a similar approach [72].

\section{Conclusion}

Over the last two decades, ADMET polymerization has been extensively studied as a unique and valuable means to access and synthesize precision polymers. These materials have provided better understanding of structure-property relationships in various systems, as they serve as models for their ill-defined commercial counterparts. While significant advances have resulted from precision polymers, many more systems have yet to be explored [73].

Open Access This article is distributed under the terms of the Creative Commons Attribution License which permits any use, distribution, and reproduction in any medium, provided the original author(s) and the source are credited.

\section{References}

1. Wagener KB, Boncella JM, Nel JG (1991) Acyclic diene metathesis (ADMET) polymerization. Macromolecules 24:2649-2657

2. Mutlu H, de Espinosa LM, Meier MAR (2011) Acyclic diene metathesis: a versatile tool for the construction of defined polymer architectures. Chem Soc Rev 40:1404-1445

3. Howard TR, Lee JB, Grubbs RH (1980) Titanium metallacarbene-metallacyclobutane reactions: stepwise metathesis. J Am Chem Soc 102:6876-6878

4. Lee JB, Gajda GJ, Schaefer WP, Howard TR, Ikariya T, Straus DA, Grubbs RH (1981) Structures of titanacyclobutanes. J Am Chem Soc 103:7358-7361

5. Lee JB, Ott KC, Grubbs RH (1982) Kinetics and stereochemistry of the titanacyclobutane-titanamethylene interconversion. Investigation of a degenerate olefin metathesis reaction. J Am Chem Soc 104:7491-7496

6. Gilliom LR, Grubbs RH (1986) A titanacyclobutane precursor to alkyl-substituted titanium-carbene complexes. Organometallics 5:721-724

7. Gilliom LR, Grubbs RH (1986) Titanacyclobutanes derived from strained cyclic olefins: the living polymerization of norbornene. J Am Chem Soc 108:733-742

8. Schaverien CJ, Dewan JC, Schrock RR (1986) A well-characterized, highly active, lewis acid free olefin metathesis catalyst. J Am Chem Soc 108:2771-2773

9. Schrock RR, DePue RT, Feldman J, Schaverien CJ, Dewan JC, Liu AH (1988) Preparation and reactivity of several alkylidene complexes of the type $\mathrm{W}\left(\mathrm{CHR}^{\prime}\right)\left(\mathrm{N}-2,6-\mathrm{C}_{6} \mathrm{H}_{3}-i-\mathrm{Pr}_{2}\right)(\mathrm{OR})_{2}$ and related tungstacyclobutane complexes. Controlling metathesis activity through the choice of alkoxide ligand. J Am Chem Soc 110:1423-1435

10. Schrock RR, DePue RT, Feldman J, Yap KB, Yang DC, Davis WM, Park L, DiMare M, Schofield M, Anhaus J, Walborsky E, Evitt E, Krüger C, Betz P (1990) Further studies of imido alkylidene complexes of tungsten, well-characterized olefin metathesis catalysts with controllable activity. Organometallics 9:2262-2275

11. Murdzek JS, Schrock RR (1987) Well-characterized olefin metathesis catalysts that contain molybdenum. Organometallics 6:1373-1374

12. Schrock RR, Murdzek JS, Bazan GC, Robbins J, DiMare M, O'Regan M (1990) Synthesis of molybdenum imido alkylidene complexes and some reactions involving acyclic olefins. J Am Chem Soc 112:3875-3886

13. Toreki R, Schrock RR (1990) A well-defined rhenium(VII) olefin metathesis catalyst. J Am Chem Soc 112:2448-2449 
14. Trnka TM, Grubbs RH (2001) The development of $\mathrm{L}_{2} \mathrm{X}_{2}=\mathrm{CHR}$ olefin metathesis catalysts: an organometallic success story. Acc Chem Res 34:8-29

15. Nguyen ST, Johnson LK, Grubbs RH, Ziller JW (1992) Ringopening metathesis polymerization (ROMP) of norbornene by a group VIII carbene complex in protic media. J Am Chem Soc 114:3974-3975

16. Nguyen ST, Grubbs RH, Ziller JW (1993) Synthesis and activities of new single-component ruthenium-based olefin metathesis catalysts. J Am Chem Soc 115:9858-9859

17. Schwab P, Grubbs RH, Ziller JW (1993) Synthesis and applications of $\mathrm{RuCl}_{2}\left(=\mathrm{CHR}^{\prime}\right)\left(\mathrm{PR}_{3}\right)_{2}$. J Am Chem Soc 118:100-110

18. Wu Z, Nguyen ST, Grubbs RH, Ziller JW (1995) Reactions of ruthenium carbenes of the type $\left(\mathrm{PPh}_{3}\right)_{2}(\mathrm{X})_{2} \mathrm{Ru}=\mathrm{CH}-\mathrm{CH}=\mathrm{CPh}_{2}$ $\left(\mathrm{X}=\mathrm{Cl}\right.$ and $\mathrm{CF}_{3} \mathrm{COO}$ ) with strained acyclic olefins and functionalized olefins. J Am Chem Soc 117:5503-5511

19. Huang J, Stevens ED, Nolan SP, Petersen JL (1999) Olefin metathesis-active ruthenium complexes bearing a nucleophilic carbene ligand. J Am Chem Soc 121:2674-2678

20. Weskamp T, Schattenmann WC, Spiegler M, Herrmann WA (1998) A novel class of ruthenium catalysts for olefin metathesis. Angew Chem Int Ed 37:2490-2493

21. Scholl M, Trnka TM, Morgan JP, Grubbs RH (1999) Increased ring closing metathesis activity of ruthenium-based olefin metathesis catalysts coordinated with imidazolin-2-ylidene ligands. Tetrahedron Lett 40:2247-2250

22. Garber SB, Kingsbury JS, Gray BL, Hoveyda AH (2000) Efficient and recyclable monomeric and dendritic Ru-based metathesis catalysts. J Am Chem Soc 122:8168-8179

23. Kingsbury JS, Harrity JPA, Bonitatebus PJ Jr, Hoveyda AH (1999) A recyclable Ru-based metathesis catalyst. J Am Chem Soc 121:791-799

24. Gessler S, Randl S, Blechert S (2000) Synthesis and metathesis reactions of a phosphine-free dihydroimidazole carbene ruthenium complex. Tetrahedron Lett 41:9973-9976

25. Vougioukalakis GC, Grubbs RH (2010) Ruthenium-based heterocyclic carbene-coordinated olefin metathesis catalysts. Chem Rev 110:1746-1787

26. Lindmark-Hamburg M, Wagener KB (1987) Acyclic metathesis polymerization. The olefin metathesis reaction of 1,5-hexadiene and 1,9-decadiene. Macromolecules 20:2949-2951

27. Opper K, Wagener KB (2011) ADMET: metathesis polycondensation. J Polym Sci Part A Polym Chem 49:821-831

28. Oskam JH, Fox HH, Yap KB, McConville DH, O'Dell R, Lichtenstein BJ, Schrock RR (1993) Ligand variation in alkylidene complexes of the type $\mathrm{Mo}(\mathrm{CHR})\left(\mathrm{NR}^{\prime}\right)\left(\mathrm{OR}^{\prime \prime}\right)_{2}$. J Organomet Chem 459:185-198

29. Wagener KB, Brzezinska K, Anderson JD, Younkin TR, Steppe K, DeBoer W (1997) Kinetics of acyclic diene metathesis (ADMET) polymerization. Influence of the negative neighboring group effect. Macromolecules 30:7363-7369

30. Lehman SE Jr, Schwendeman JE, O'Donnell PM, Wagener KB (2003) Olefin isomerization promoted by olefin metathesis catalysts. Inorg Chim Acta 345:190-198

31. Courchay FC, Sworen JC, Wagener KB (2003) Metathesis activity and stability of new generation ruthenium polymerization catalysts. Macromolecules 36:8231-8239

32. Fokou PA, Meier MAR (2010) Studying and suppressing olefin isomerization side reactions during ADMET polymerizations. Macromol Rapid Commun 31:368-373

33. Hong SH, Sanders DP, Lee CW, Grubbs RH (2005) Prevention of undesirable isomerization during olefin metathesis. J Am Chem Soc 127:17160-17161

34. Simocko C, Wagener KB (2013) Effects of boron-containing lewis acids on olefin metathesis. Organometallics 32:2513-2516
35. Weychardt H, Plenio H (2008) Acyclic diene metathesis polymerization of divinylarenes and divinylferrocenes with Grubbstype olefin metathesis catalysts. Organometallics 27:1479-1485

36. Simocko C, Yang Y, Swager TM, Wagener KB (2013) Metathesis step-growth polymerization in ionic liquids. ACS Macro Lett 2:1061-1064

37. Knuuttila H, Lehtinen A, Nummila-Pakarinen A (2004) Advanced polyethylene technologies-controlled material properties, long-term properties of polyolefins. Adv Polym Sci 169:13-27

38. Fawcet EW, Gibson RQ, Perrin MH, Patton JG, Williams EG (1937) Imperial Chemical Industries Ltd. Pat 2, pp 816-883

39. Baughman TW, Wagener KB (2005) Recent advances in ADMET polymerization. In: Buchmeiser M (ed). Springer, Berlin, pp $1-42$

40. Rojas G, Wagener KB (2008) Avoiding olefin isomerization during decyanation of alkylcyano $\alpha$, $\omega$-dienes: a deuterium labeling and structural study of mechanism. J Org Chem 73:4962-4970

41. Rojas G, Wagener KB (2009) Precisely and irregularly sequenced ethylene/1-hexene copolymers: a synthesis and thermal study. Macromolecules 42:1934-1947

42. Hosoda S, Nozue Y, Kawashima Y, Suita K, Seno S, Nagamatsu T, Wagener KB, Inci B, Zuluaga F, Rojas G, Leonard JK (2011) Effect of the sequence length distribution on the lamellar crystal thickness and thickness distribution of polyethylene: perfectly equisequential ADMET polyethylene vs ethylene/ $\alpha$-olefin copolymer. Macromolecules 44:313-319

43. Hosoda S, Nozue Y, Kawashima Y, Utsumi S, Nagamatsu T, Wagener K, Berda E, Rojas G, Baughman T, Leonard J (2009) Perfectly controlled lamella thickness and thickness distribution: a morphological study on ADMET polyolefins. Macromol Symp 282:50-64

44. Rojas G, Inci B, Wei Y, Wagener KB (2009) Precision polyethylene: changes in morphology as a function of alkyl branch size. J Am Chem Soc 131:17376-17386

45. Inci B, Lieberwirth I, Steffen W, Mezger M, Graf R, Landfester K, Wagener KB (2012) Decreasing the alkyl branch frequency in precision polyethylene: effect of alkyl branch size on nanoscale morphology. Macromolecules 45:3367-3376

46. Inci B, Wagener KB (2011) Decreasing the alkyl branch frequency in precision polyethylene: pushing the limits toward longer run lengths. J Am Chem Soc 133:11872-11875

47. Qiu W, Sworen J, Pyda M, Nowak-Pyda E, Habenschuss A, Wagener KB, Wunderlich B (2006) Effect of the precise branching of polyethylene at each $21 \mathrm{st} \mathrm{CH}_{2}$ group on its phase transitions, crystal structure, and morphology. Macromolecules 39:204-217

48. Lieser G, Wegner G, Smith JA, Wagener KB (2004) Morphology and packing behavior of model ethylene/propylene copolymers with precise methyl branch placement. Colloid Polym Sci 282:773-781

49. Matsui K, Seno S, Nozue Y, Shinohara Y, Amemiya Y, Berda EB, Rojas G, Wagener KB (2013) Influence of branch incorporation into the lamella crystal on the crystallization behavior of polyethylene with precisely spaced branches. Macromolecules 46:4438-4446

50. Nozue Y, Kawashima Y, Seno S, Nagamatsu T, Hosoda S, Berda EB, Rojas G, Baughman TW, Wagener KB (2011) Unusual crystallization behavior of polyethylene having precisely spaced branches. Macromolecules 44:4030-4034

51. Bowers CR, Wei Y, Aitken BS, Reeg CR, Akel CD (2012) Molecular dynamics in precision deuteriomethyl branched polyethylene from solid-state deuterium NMR. Polymer 53:2633-2642

52. Wei Y, Graf R, Sworen JC, Cheng C-Y, Bowers CR, Wagener KB, Spiess HW (2009) Local and collective motions in precise 
polyolefins with alkyl branches: a combination of ${ }^{2} \mathrm{H}$ and ${ }^{13} \mathrm{C}$ solidstate NMR spectroscopy. Angew Chem Int Ed 48:4617-4620

53. Spiess WH (1983) Molecular dynamics of solid polymers as revealed by deuteron NMR. Colloid Polym Sci 261:193-209

54. Zutty NL, Faucher JA, Bonotto S, Mark HF (eds) (1967) Encyclopedia of polymers science and technology. Wiley, New York

55. Odian G (1991) Principles of polymerization, 3rd edn. Wiley, New York, p 460

56. Watson MD, Wagener KB (2000) Ethylene/vinyl acetate copolymers via acyclic diene metathesis polymerization. Examining the effect of "long" precise ethylene run lengths. Macromolecules 33:5411-5417

57. Baughman TW, Chan CD, Winey KI, Wagener KB (2007) Synthesis and morphology of well-defined poly(ethylene-coacrylic acid) copolymers. Macromolecules 40:6564-6571

58. Seitz ME, Chan CD, Opper KL et al (2010) Nanoscale morphology in precisely sequenced poly(ethylene-co-acrylic acid) zinc ionomers. J Am Chem Soc 132:8165-8174

59. Boz E, Ghiviriga I, Nemeth AJ, Jeon K, Alamo RG, Wagener KB (2008) Random, defect-free ethylene/vinyl halide model copolymers via condensation polymerization. Macromolecules 41:25-30

60. Opper KL, Wagener KB (2009) Precision sulfonic acid ester copolymers. Macromol Rapid Commun 30:915-919

61. Opper KL, Markova D, Klapper M, Müllen K, Wagener KB (2010) Precision phosphonic acid functionalized polyolefin architectures. Macromolecules 43:3690-3698

62. Markova D, Opper KL, Wagner M, Klapper M, Wagener KB, Müllen K (2013) Synthesis of proton conducting phosphonic acid-functionalized polyolefins by the combination of ATRP and ADMET. Polym Chem 4:1351-1363

63. Sydlik SA, Delgado PA, Inomata S et al (2013) Triptycenecontaining polyetherolefins via acyclic diene metathesis polymerization. J Polym Sci Part A Polym Chem 51:1695-1706
64. Matloka PP, Wagener KB (2006) The acyclic diene metathesis (ADMET) polymerization approach to silicon containing materials. J Mol Catal A Chem 257:89-98

65. Leonard JK, Wei Y, Wagener KB (2012) Synthesis and thermal characterization of precision poly(ethylene-co-vinyl amine) copolymers. Macromolecules 45:671-680

66. Marmo JC, Wagener KB (1993) Acyclic diene metathesis (ADMET) depolymerization. Synthesis of mass-exact telechelic polybutadiene oligomers. Macromolecules 26:2137-2138

67. Marmo JC, Wagener KB (1995) ADMET depolymerization. Synthesis of perfectly difunctional $(f=2.0)$ telechelic polybutadiene oligomers. Macromolecules 28:2602-2606

68. Schulz MD, Ford RR, Wagener KB (2013) Insertion metathesis depolymerization. Polym Chem 4:3656-3658

69. Tao D, Wagener KB (1993) Acyclic diene metathesis (ADMET) polymerization. Synthesis of telechelic polyacetylene. Polym Prepr 34:469-470

70. Schwendeman JE, Wagener KB (2009) Synthesis of amorphous hydrophobic telechelic hydrocarbon diols via ADMET polymerization. Macromol Chem Phys 210:1818-1833

71. Tamura H, Nakayama A (2002) Functional telechelic polymer synthesis via ADMET polymerization. J Macromol Sci Pure Appl Chem A 39:745-758

72. Tamura H, Maeda N, Matsumoto R, Nakayama A, Hayashi H, Ikushima K, Kuraya M (1999) Synthesis of ester terminated telechelic polymer via ADMET polymerization. J Macromol Sci Pure Appl Chem A 36:1153-1170

73. Atallah P, Wagener KB, Schulz MD (2013) ADMET: the future revealed. Macromolecules 46:4735-4741 